\title{
Correlation Between MRI Findings and Histological Diagnosis of Brainstem Glioma
}

\author{
Luxin Yin, Liwei Zhang
}

\begin{abstract}
Objective: In most studies, treatment decisions of brainstem glioma are based solely on MRI features and do not incorporate a histopathological diagnosis. In the current study, we sought to compare MRI characteristics with histopathological findings of bainstem glioma. Methods: From April 2003 through April 2012, 150 patients were diagnosed with brainstem gliomas by MRI and microsurgically treated in Tiantan Hospital, Beijing, China. All the MRI and histopathological findings of these patients were respectively reviewed. Results: Of the 150 patients, 65 were female and 85 were male, 120 were adults and 30 were children (age < 18 years), 108 were low-grade glioma (72.0\%), 35 were high-grade glioma (23.3\%). The accuracy of the MRI diagnosis for brainstem glioma was $95.3 \%$. Data analysis of the MRI findings revealed that a focal lesion was associated with a more favorable histopathological diagnosis in intrinsic $(\mathrm{P}=0.005)$ and exophytic $(\mathrm{P}=0.001)$ brainstem glioma patients. In the intrinsic diffuse type, tumors without enhancement had more favorable pathological findings $(\mathrm{P}=0.009)$. Conclusions: To our knowledge, this is the largest case series of this nature reported in the literature to date. The results of this study suggest that MRI features of brainstem gliomas could predict some pathological features and guide prognosis, choice of biopsy and treatment modalities. The pathology of tumors with a focal appearance on MRI was associated with a prognosis that was significantly better than their diffuse counterparts. For the intrinsic diffuse gliomas, non-enhancing tumors had pathology suggestive of a favorable prognosis.
\end{abstract}

RÉSUMÉ: Corrélation entre les constatations à l'IRM et le diagnostic anatomopathologique des gliomes du tronc cérébral. Objectif : Dans la plupart des études, les décisions de traitement des gliomes du tronc cérébral sont fondées uniquement sur les caractéristiques de la tumeur à l'IRM et n'incluent pas le diagnostic anatomopathologique. Dans cette étude, nous avons comparé les caractéristiques de gliomes du tronc cérébral à l'IRM avec les constatations anatomopathologiques au moment de la chirurgie. Méthode : Un diagnostic de gliome du tronc cérébral a été posé à l'IRM chez 150 patients entre avril 2003 et avril 2012 et ils ont été traités à l'Hôpital Tiantan, à Beijing, en Chine. Nous avons revu toutes les constatations faites à l'IRM et en anatomopathologie chez ces patients. Résultats : Parmi les 150 patients, 65 étaient des femmes et 85 étaient des hommes, 120 étaient des adultes et 30 étaient des enfants de moins de 18 ans, et 108 étaient porteurs de gliomes de bas grade de malignité (70,0\%) et 35 étaient porteurs de gliomes de haut grade de malignité $(23,3 \%)$. L'exactitude du diagnostic de gliome du tronc cérébral à l'IRM était de 95,3\%. L'analyse des constatations faites à l'IRM a montré qu'une lésion focale était associée à un diagnostic anatomopathologique plus favorable chez les patients atteints de gliomes du tronc cérébral intrinsèques $(\mathrm{p}=0,005)$ et exophytiques $(\mathrm{p}=0,001)$. Parmi les tumeurs intrinsèques de type diffus, les tumeurs sans rehaussement avaient des caractéristiques anatomopathologiques plus favorables $(\mathrm{p}=0,009)$. Conclusions : À notre connaissance, ceci constitue la plus grande série de cas de cette nature rapportée dans la littérature à ce jour. Selon les résultats de cette étude, les caractéristiques des gliomes du tronc cérébral à l'IRM pourraient prédire certaines caractéristiques anatomopathologiques et guider le pronostic, le choix de biopsie et les modalités de traitement. La pathologie des tumeurs ayant un aspect focal à l'IRM était significativement meilleure que celle des tumeurs dont l'aspect était diffus. Dans le cas des gliomes diffus intrinsèques, les tumeurs non rehaussantes avaient des caractéristiques anatomopathologiques plus favorables.

Can J Neurol Sci. 2013; 40: 348-354

Brainstem gliomas can occur at any age, although they generally present in childhood, with a mean age of diagnosis of 7-9 years ${ }^{1-4}$. In most studies, treatment decisions are based solely on magnetic resonance imaging (MRI) features and do not incorporate a histopathological diagnosis. Most authors consider imaging methods to be sufficiently reliable ${ }^{1,2}$. Thus, the effect of MRI findings on treatment decisions for brainstem tumors is very high, but the accuracy of MRI-based diagnosis of brainstem gliomas has not been conclusively verified by histopathological finding $\mathrm{s}^{2}$. In the current study, we compared MRI characteristics with histopathological findings of brainstem lesions.

\section{Patients and Methods}

From April 2003 through April 2012, 150 patients were diagnosed with brainstem gliomas by MRI and biopsied or resected, wholly or in part in Tiantan Hospital, Beijing, China.

\footnotetext{
From the Department of Neurosurgery, Beijing Tiantan Hospital, Capital Medical University, Beijing, PR China.

Received May 31, 2012. Final Revisions Submitted November 14, 2012. Correspondence to: Li Wei Zhang, Department of Neurosurgery, Beijing Tiantan Hospital, Capital Medical University, TiantanXili 6, Chongwen District, Beijing, 100050, PR China. Email: yinluxin530@163.com.
} 

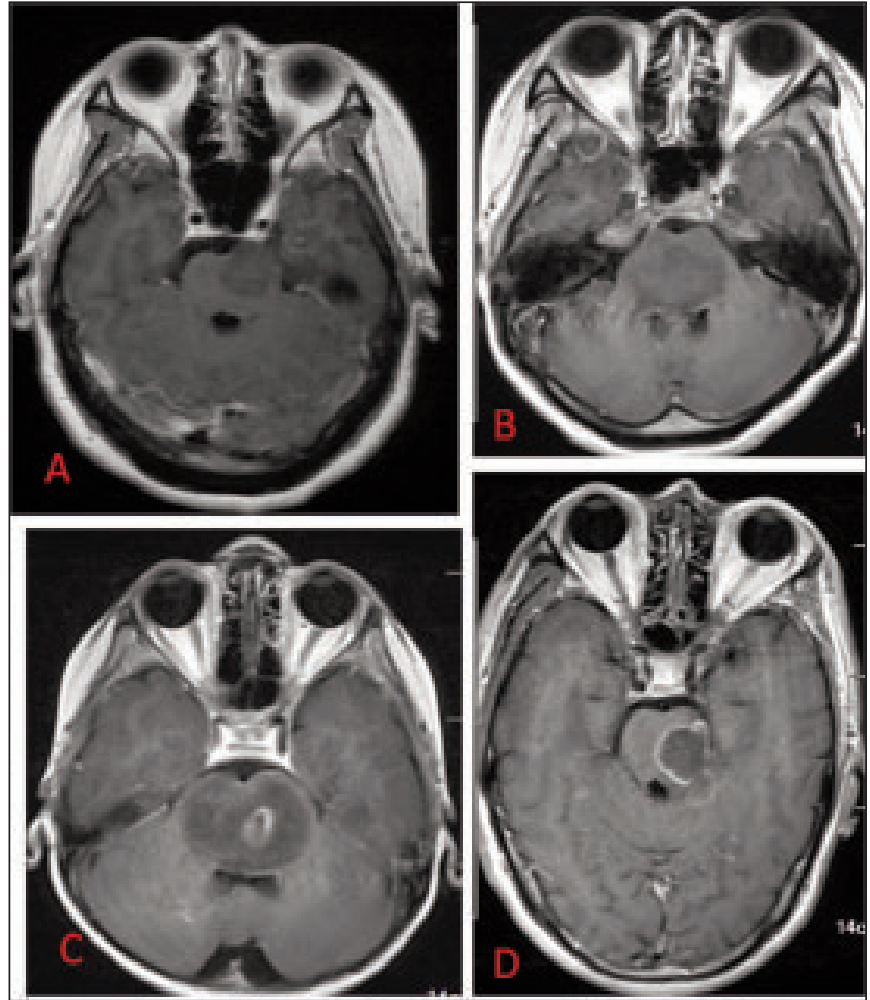

Figure 1: A. focal intrinsic nonenhancing type. B. diffuse intrinsic nonenhancing type. C. diffuse intrinsic enhancing type. D. focal intrinsic enhancing type.

Of the 150 patients, 65 were female and 85 were male, 120 were adults and 30 were children (age $<18$ years), with a mean age of 32 years (range, 6-58 years). Patients were followed up from 14 days to 110 months after surgery (mean, 29.4 months, median, 34 months).
All MRIs were reviewed and independently classified in a blinded fashion by two neurosurgeons (Luxin Yin and Liwei Zhang) with special interest in brainstem gliomas. The pathology diagnosis was made by at least two senior pathologists using the World Health Organization (WHO) classification.

Brainstem glioma can be classified, according to Choux et $\mathrm{al}^{3}$, as: (1) diffuse intrinsic or exophytic; (2) focal intrinsic or exophytic, cystic or solid, located in the medulla, pons, or midbrain; (3) dorsal or lateral exophytic, arising in the subependymal zone and growing into the fourth ventricle; and (4) cervicomedullary, not including cervical spinal cord tumors that respect the border of the medulla.

According to the classification, we segregated the tumors into three groups: (1) intrinsic type; (2) exophytic type and (3) cervicomedullary. In addition, we grouped tumors into enhancing or nonenhancing as well as diffuse or focal. Focal tumors were said to have well-defined margins on MRI and occupy less than $50 \%$ of the axial diameter of the brainstem, whereas diffuse tumors have poorly defined margins and occupy more than $50 \%$ of the axial diameter of the brainstem ${ }^{1}$.

Intrinsic brainstem lesions were separated by MRI findings into four groups: (1) diffuse intrinsic enhancing lesions (MRI imaging showing a diffuse hypointense lesion on T1-weighted imaging; contrast enhancing lesion on T1-weighted imaging; and diffuse hyperintense lesion on T2-weighted imaging); (2) diffuse intrinsic nonenhancing lesions (MRI imaging showing a diffuse hypointense lesion on T1-weighted imaging; noncontrast enhancing lesion on T1-weighted imaging; and diffuse hyperintense lesion on T2-weighted imaging); (3) focal intrinsic enhancing lesions (MRI imaging showing a focal hypointense lesion on T1-weighted imaging; contrast-enhancing lesion on T1-weighted imaging; and focal hyperintense lesion on T2weighted imaging); (4) focal intrinsic nonenhancing lesions (MRI imaging showing a focal hypointense lesion on T1weighted imaging; noncontrast-enhancing lesion on T1weighted imaging; and focal hyperintense lesion on T2-weighted imaging).
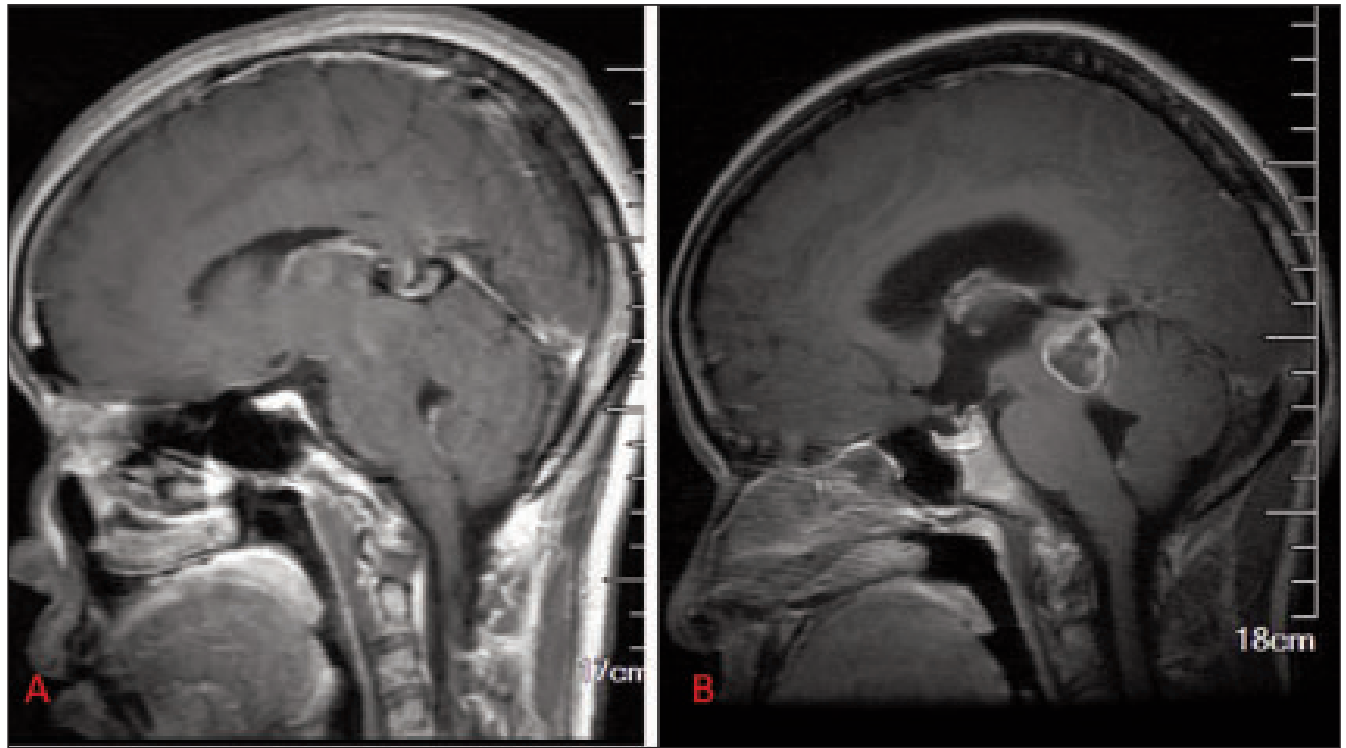

Figure 2: Tectal glioma of the focal exophytic type A. tectal glioma without enhancement. B. tectal glioma with enhancement. 

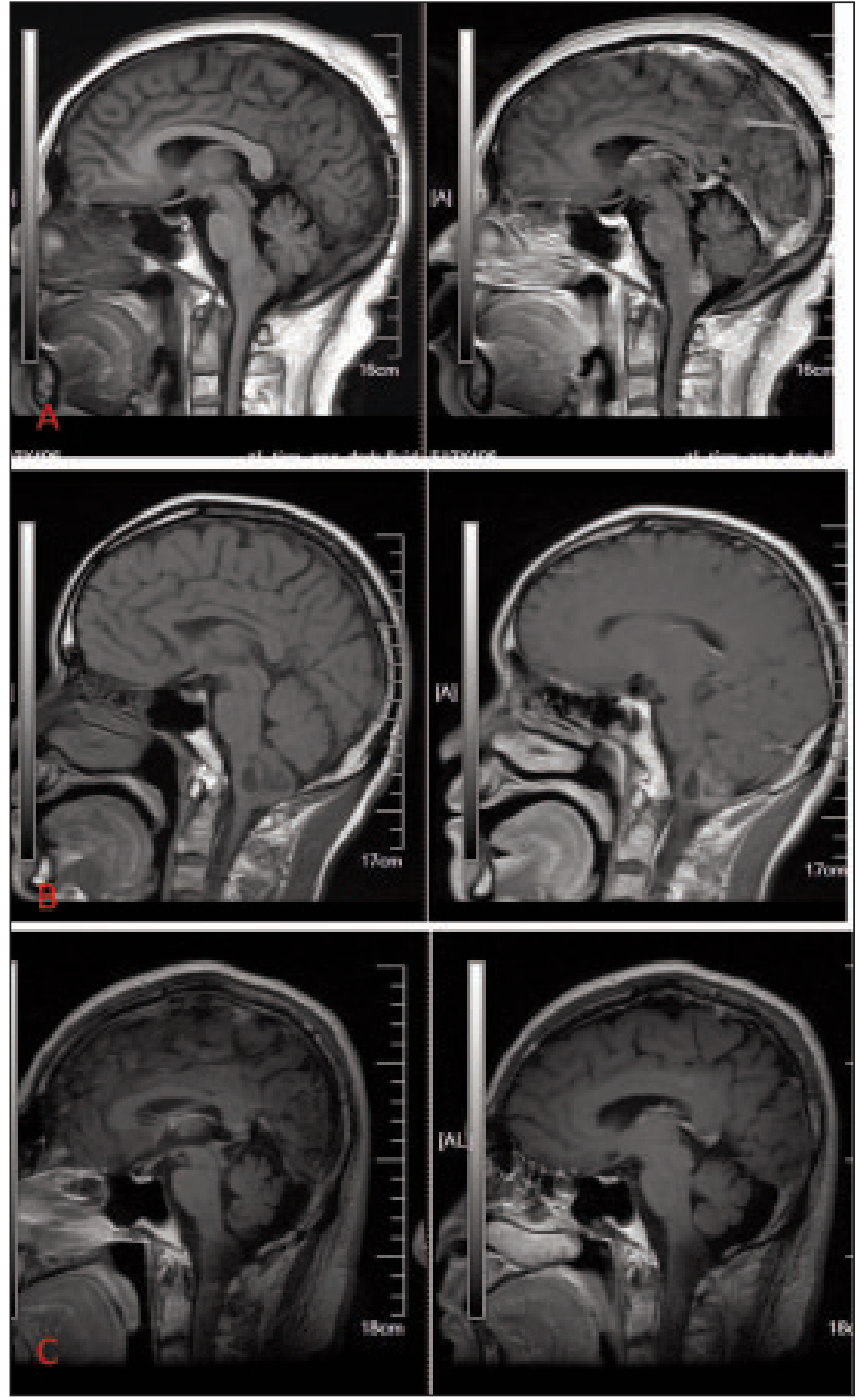

Figure 3: Dorsal exophytic tumors of the medulla, a type of focal exophytic tumor. A. dorsal exophytic tumors of the medulla with enhancement. B. dorsal exophytic tumors of the medulla with enhancement and cystic changes. $C$. dorsal exophytic tumors of the medulla without enhancement or cystic changes.
Exophytic brainstem lesions were also separated into four groups by MRI findings: (1) diffuse exophytic enhancing lesions (MRI imaging showing a diffuse hypointense lesion on T1weighted imaging which invaded the surrounding structures; contrast-enhancing lesion on T1-weighted imaging; and diffuse hyperintense lesion on T2-weighted imaging); (2) diffuse exophytic nonenhancing lesions (MRI imaging showing a diffuse hypointense lesion on T1-weighted imaging which invaded the surrounding structures; noncontrast enhancing lesion on T1-weighted imaging; and diffuse hyperintense lesion on T2-weighted imaging); (3) focal exophytic enhancing lesions (MRI imaging showing a focal hypointense lesion on T1weighted imaging not totally confined within the brainstem; contrast-enhancing lesion on T1-weighted imaging; and focal hyperintense lesion on T2-weighted imaging); (4) focal exophytic nonenhancing lesions (MRI imaging showing a focal hypointense lesion on T1-weighted imaging not totally confined within the brainstem; noncontrast enhancing lesion on T1weighted imaging; and focal hyperintense lesion on T2-weighted imaging). Cervicomedullary type tumors were described as a single group (Figures 1-5).

Low grade glioma was defined as a group which was composed of the pilocytic astrocytoma (WHOI), ganglioglioma (WHOI), ependymoma (WHOII), astrocytoma (WHOII) and oligodendroglioma (WHOII); High grade glioma was defined as a group which was composed of anaplastic gliomas (WHOIII) and glioblastoma (WHOIV).

All subjects or their legal representative gave written informed consent to the study protocol, which was approved by the ethics committee of Beijing Tiantan hospital.

\section{Surgical Technique}

Following the induction of general anesthesia, 75 patients underwent total resection, 23 a near-total resection ( $>90 \%), 37$ a subtotal resection (50-90\%), ten a partial resection (25-50\%) and five a biopsy. Of the 150 patients, 75 received the midline approach, 30 received the temporal-occipital approach, 25 received the parietal-occipital approach, 15 patients received the retrosigmoid approach, 5 received the far lateral approach. All biopsy specimens were formalin-fixed and analyzed after

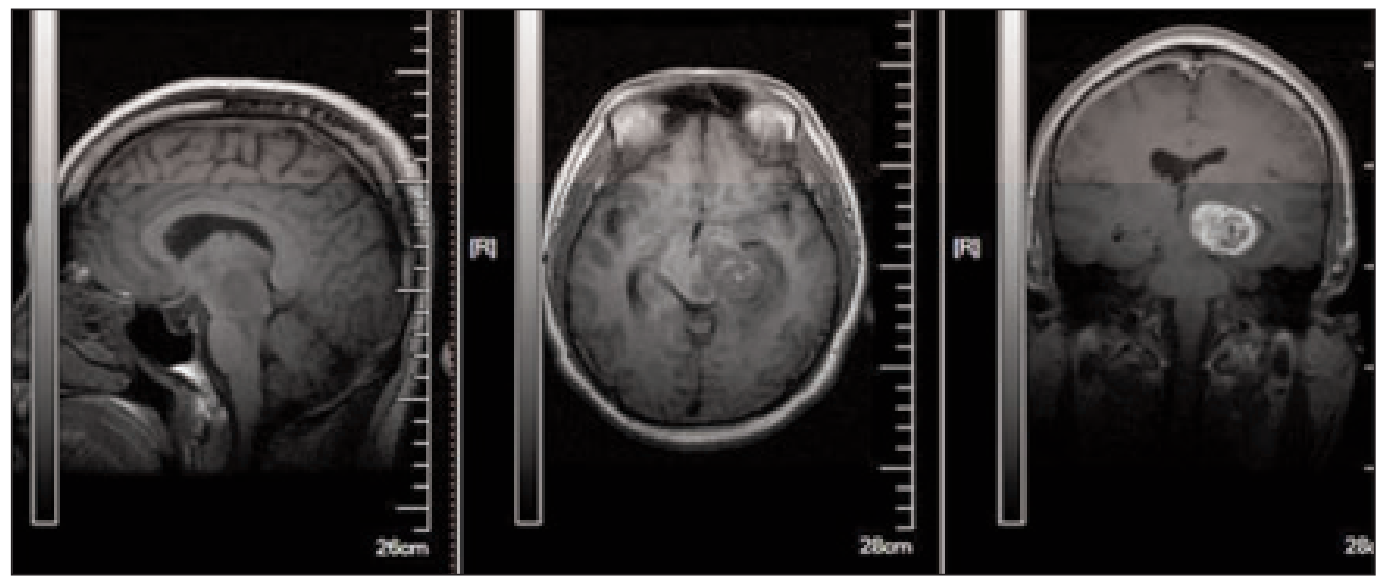

Figure 4: Diffuse exophytic enhancing type. 


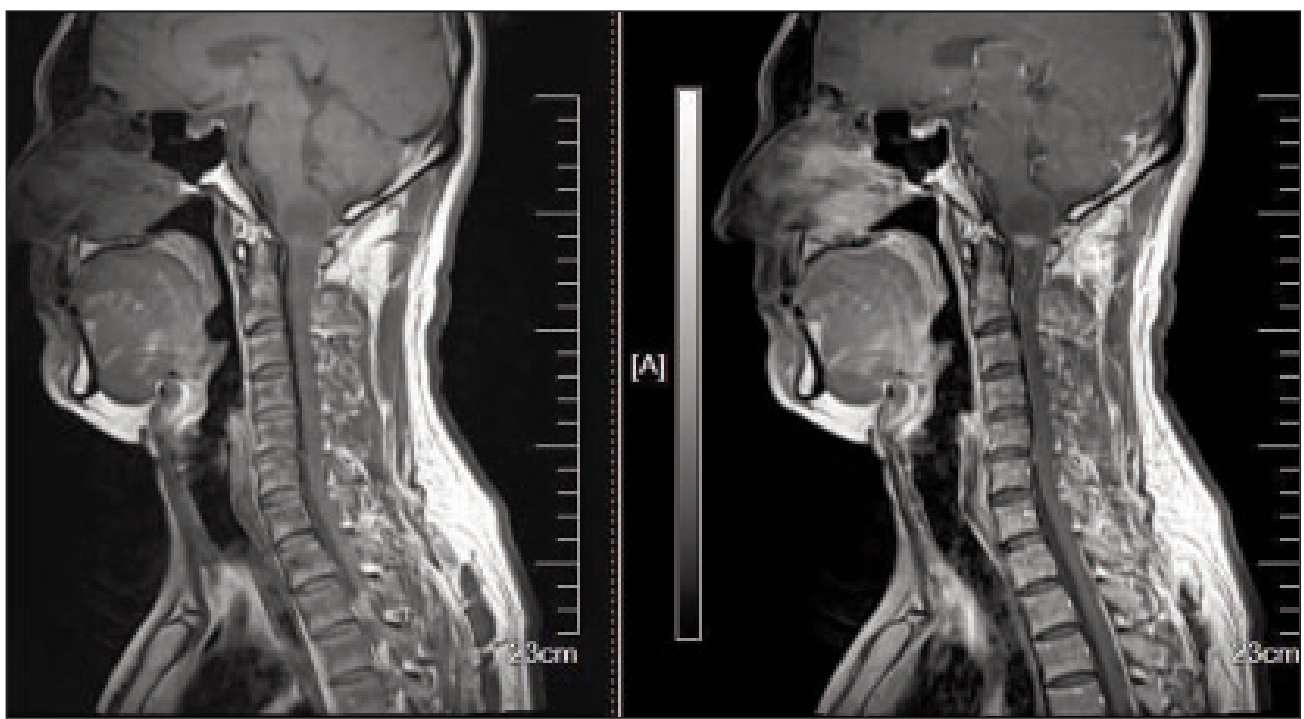

Figure 5: Cervicomedullary lesions with enhancement and cystic changes.

staining with hematoxylin and eosin, Masson trichrome, and immunohistochemistry.

\section{Data Analysis and Statistical Analysis}

Data analysis was performed using SPSS 13.0, Cross-table analysis of the following variables was performed with regard to the radiological findings and histological diagnosis: aspect in T1 diffuse versus focal and enhanced versus nonenhanced contrast. All lesions in T2 were hyperintense. Cross-table analysis of the adults and children (age $<18$ years) was performed in both the intrinsic and exophytic groups.

\section{ReSUlts}

On the radiological findings, 92 patients had intrinsic lesions. Of these, 37 (including eight children) had diffuse intrinsic enhancing brainstem lesions, 9 (including two children) had diffuse intrinsic nonenhancing brainstem lesions, 13 (including three children) had focal intrinsic nonenhancing brainstem lesions, and 33 (including six children) had focal intrinsic enhancing brainstem lesions (Table 1).
Fifty-five patients had exophytic brainstem lesions. Of these, 18 (including six children) had diffuse exophytic enhancing brainstem lesions, 27 (including two children) had focal exophytic enhancing brainstem lesions and 10 (including three children) had focal exophytic nonenhancing brainstem lesions (Table 2).

Three patients had cervicomedullary lesions. There were no diffuse exophytic nonenhancing brainstem lesions.

Neurologically, the preoperative symptoms of the patients may be transiently or permanently worsened after surgery (about $10 \%$ in our ward). The incidence of postoperative neurological deficit was about $40 \%$. Further neurological injury including respiratory arrest, which is usually permanent, were more common following surgery for medullary lesions.

\section{Correlation of Histological and Radiological Findings}

Of the 150 patients, 65 were female and 85 were male, 108 were low-grade glioma $(72.0 \%), 35$ were high-grade glioma (23.3\%), 7 revealed other pathologies (two cases of reactive

Table 1: Histological diagnosis in the patients with intrinsic brainstem glioma

\begin{tabular}{|c|c|c|c|c|c|c|c|c|}
\hline & Histology & Pilocytic astrocytoma & Ganglioglioma & Ependymocytoma & Astrocytoma & Oligodendroglioma & Anaplastic glioma & Glioblastoma \\
\hline Diffuse intrinsic & Adults & 0 & 1 & 0 & 11 & 0 & 7 & 6 \\
\hline enhancing type & Children & 0 & 0 & 0 & 6 & 0 & 2 & 0 \\
\hline Diffuse intrinsic & Adults & 0 & 0 & 0 & 5 & 2 & 0 & 0 \\
\hline 1onenhancing type & Children & 0 & 0 & 0 & 2 & 0 & 0 & 0 \\
\hline Focal intrinsic & Adults & 0 & 0 & 0 & 16 & 4 & 3 & 1 \\
\hline enhancing type & Children & 1 & 0 & 0 & 5 & 0 & 0 & 0 \\
\hline Focal intrinsic & Adults & 0 & 0 & 0 & 10 & 0 & 0 & 0 \\
\hline lonenhancing type & Children & 1 & 0 & 0 & 1 & 0 & 1 & 0 \\
\hline
\end{tabular}


Table 2: Histological diagnosis in the patients with exophytic brainstem glioma

\begin{tabular}{|c|c|c|c|c|c|c|c|c|}
\hline \multirow{3}{*}{$\begin{array}{c}\text { Diffuse exophytic } \\
\text { enhancing type }\end{array}$} & Histology & Pilocytic astrocytoma & Ganglioglioma & Ependymoma & Astrocytoma & Oligodendroglioma & Anaplastic glioma & Glioblastoma \\
\hline & Adults & 1 & 0 & 0 & 0 & 3 & 6 & 2 \\
\hline & Children & 0 & 0 & 0 & 1 & 1 & 1 & 3 \\
\hline \multirow{2}{*}{$\begin{array}{l}\text { Focal exophytic } \\
\text { nonenhancing type }\end{array}$} & Adults & 1 & 0 & 0 & 6 & 0 & 0 & 0 \\
\hline & Children & 1 & 0 & 0 & 2 & 0 & 0 & 0 \\
\hline \multirow{2}{*}{$\begin{array}{l}\text { Focal exophytic } \\
\text { enhancing type }\end{array}$} & Adults & 2 & 0 & 5 & 13 & 2 & 2 & 1 \\
\hline & Children & 0 & 0 & 0 & 2 & 0 & 0 & 0 \\
\hline
\end{tabular}

astrogliosis, two metastases, two cases of melanoma and one epidermoid cyst). The accuracy of the MRI diagnosis for brainstem glioma was therefore $95.3 \%$ (see Chart).

Histological evaluation revealed low grade glioma in all of the nine patients with diffuse intrinsic nonenhancing brainstem lesions. Gliomas were verified in $33(89.2 \%)$ of the 37 patients showing diffuse enhancing brainstem lesions. Of these 33 patients, 17 had low-grade gliomas and 16 had high-grade gliomas. Four patients had pathologies other than glioma (two melanomas and two examples of reactive astrogliosis).

In the cases involving focal intrinsic nonenhancing brainstem lesions, a diagnosis of brainstem glioma was achieved in 12 patients, of which only one was high grade. In the 33 patients involving focal intrinsic enhancing brainstem lesions, a diagnosis of brainstem glioma was verified in 30 (90.9\%), of which 26 were low-grade and four were high-grade. Pathologies different from glioma were diagnosed in three patients (all adults), of whom two had metastases and one had an epidermoid cyst.

Histological evaluation revealed brainstem glioma in all of the 18 patients with diffuse exophytic enhancing brainstem lesions. Six cases were low-grade, 12 cases were high-grade. There were no examples of a diffuse exophytic nonenhancing brainstem lesion.

In the cases involving focal exophytic nonenhancing brainstem lesions, diagnosis of brainstem glioma was confirmed in ten patients (100\%), all of whom had low-grade gliomas. In the 27 cases involving focal exophytic enhancing brainstem lesions, a diagnosis of brainstem glioma was verified in 27 patients $(100 \%)$, of whom 24 had low-grade glioma and three had high-grade gliomas. There were five ependymomas, all of which were dorsal, enhancing, exophytic, cystic and arose from the medulla.

All three cervicomedullary lesions were ependymomas.

Data analysis revealed that having a focal lesion on MRI was associated with more favorable pathology in intrinsic $(\mathrm{P}=0.005)$ and exophytic $(\mathrm{P}=0.001)$ brainstem gliomas. In the intrinsic diffuse type, the absence of contrast enhancement was a significant factor associated with more favorable pathology $(\mathrm{P}=0.009)$.

Our results suggest that younger age is not associated with more favorable histopathology whether intrinsic $(\mathrm{P}=0.624)$ or exophytic $(\mathrm{P}=0.771)$.

\section{Discussion}

\section{Focal intrinsic brainstem glioma}

Focal intrinsic brainstem gliomas are defined as demarcated lesions of the brainstem found in the midbrain, pons or medulla. These focal tumors may be solid or cystic and they always have clear, distinct borders on MRI. The tumor size and characteristics are similar on both $\mathrm{T} 1$ and $\mathrm{T} 2$ sequences because of the relative lack of infiltration and edema. Tumor enhancement following gadolinium administration may be variable. These focal tumors are mostly histologically benign lesions ${ }^{4}$. In our series, there were 46 patients with intrinsic focal lesions, of which 38 were low grade glioma $(82.6 \%)$. Our study also showed that among those with focal enhancing brainstem lesions, a diagnosis of glioma was achieved in only $88.9 \%$ of adult cases. Histological diagnosis revealed a variety of other pathologies including metastatic carcinoma and epidermoid cyst. Zagzag et $\mathrm{al}^{5}$ reported seven cases of primitive neuroectodermal tumors of the brainstem. Marcos Dellaretti et $\mathrm{al}^{6}$ found that only $34.7 \%$ were correctly diagnosed as glioma in such MRI patterns. That is to

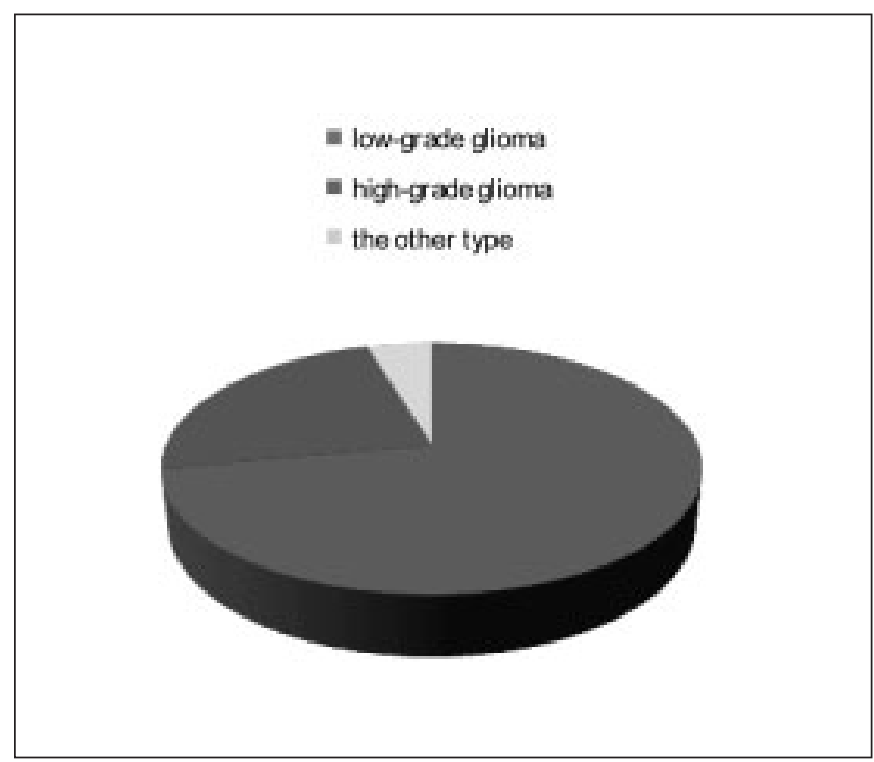

Chart: Pathology of the 150 brainstem glioma patients diagnosed by MRI before operation. 
say, such MRI patterns may be not sufficiently specific and alternative diagnoses should be considered for which management varies widely. A tissue diagnosis is optimal.

\section{Diffuse intrinsic brainstem glioma}

The most typical brainstem glioma, accounting for $80 \%$ of cases, also has the worst prognosis of any brain tumor in children $^{7-9}$. Onset usually occurs between the ages of seven and nine years. Typically, intrinsic diffuse gliomas appear as enlarging tumors of the pons with involvement of an entire cross section of the brain stem in T2 weighted signal images. Tumors often exhibit a ventral exophytic component with basilar artery engulfment. After gadolinium infusion, the tumors may slightly enhance, usually in an inhomogeneous fashion. This finding has not previously been shown to have any prognostic significance ${ }^{10,11}$. However, in the present study, we found that tumors without enhancement have more favorable pathology. Of our intrinsic diffuse enhancing brain stem gliomas $40.5 \%$ were high grade while all the nine intrinsic diffuse nonenhancing gliomas were low grade. Our study also showed that intrinsic diffuse brainstem lesions did have a worse pathological diagnosis than focal lesions $(\mathrm{P}=0.005)$.

\section{Focal exophytic brainstem glioma}

The cases of focal exophytic brainstem lesions were divided into three groups as tectal glioma, exophytic medulla glioma and a other type. All the tumors of the focal exophytic type which were not tectal tumors or dorsal exophytic tumors of medulla were classified into the other type.

Focal tectal glioma (located in the posterior part of the midbrain, behind the aqueduct of Sylvius) (Figure 2 (A-B)) is a well defined clinical entity with particularly good prognosis. It is a rare tumor, accounting for less than $5 \%$ of brainstem gliomas and in these cases, histology usually shows a low-grade astrocytoma $^{12-18}$. Since the clinical course of these tumors is usually indolent, open surgery is not a preferred option. However, of our three enhancing tectal gliomas, two were highgrade. For the patients whose tumors evolve from non-enhancing to enhancing, the pathology may have changed from low to high grade. Such tumors may be biologically different from indolent examples. Regardless, surgical resection and pathological diagnosis of all enhancing tectal tumors may be the best choice for the patients although this remains controversial. Partial resection may prolong survival and facilitate subsequent complementary treatment ${ }^{19}$.

Dorsal exophytic tumors of the medulla (Figure 3 (A-C)) was another category. Tumors involving the medulla may have lower cranial nerve deficits with speech and swallowing difficulties and limb weakness. We found that most of the dorsal exophytic enhancing and cystic tumors of medulla were ependymomas while most of the noncystic lesions were astrocytomas.

In general, focal exophytic tumors share four characteristics: (1) long duration of symptoms before diagnosis; (2) low grade histology (most of them are astrocytomas); (3) amenable to surgical resection in many cases; and (4) long-term survival ${ }^{1}$.

\section{Diffuse exophytic brainstem glioma}

Diffuse exophytic glioma (Figure 4) is defined as a brainstem glioma which invades neighbouring structures such as thalamus and cerebellum. In our series, all cases enhanced, most of which $(66.7 \%)$ were high-grade (five were glioblastoma, seven were anaplastic gliomas) while only $40.5 \%$ of our diffuse intrinsic enhancing brainstem tumors were high-grade. The pathology of tumors in this category was the least favorable of all categories in the present study.

\section{Cervicomedullary lesions}

Cervicomedullary brainstem gliomas (Figure 5) are similar to intramedullary spinal cord gliomas. The epicenter of these tumors may be either in the medulla or cervical spinal cord. On MRI, these tumors show mixed low and intermediate signal intensity within the solid part of the tumor. Magnetic resonance imaging assists in delineating the rostral and caudal poles of these tumors, as well as identifying any associated cysts. The majority of these tumors are benign low-grade gliomas, and they demonstrate distinct growth patterns ${ }^{20,21}$. In our cases, 3 cervicomedullary lesions were all cystic and ependymomas. Cystic degeneration may be a sign of ependymomas in this category.

\section{Children and adults}

It has been suggested that adult brainstem glioma patients have a better prognosis than children, suggesting a different biological behavior between these two age groups ${ }^{1,4,7-9}$. In our series, we found that there was no significant difference of the pathology between these two age groups no matter what the intrinsic type or in the exophytic type.

Liu et $\mathrm{a}^{22}$ injected the C 6 cell line into the pons of Wistar rats and found that relatively focal tumors were observed in adult rats while diffusive tumors were found in juvenile rats. He hypothesized that the different growth pattern and invasiveness of brainstem glioma between children and adult could be due to the different host cellular environments between the two age groups. Further investigation of the different host-responses between childhood and adult brainstem glioma is needed.

\section{Conclusions}

To our knowledge, this is the largest case series of this nature reported in the literature to date. The results of this study may serve to better predict the pathology from MRI findings and help to obviate the need for invasive procedures. Further studies will include follow-up with these patients to extend our understanding of the correlation between MRI findings, histopathology and outcomes. 


\section{REFERENCES}

1. Guillamo JS, Doz F, Delattre JY. Brain stem gliomas. Curr Opin Neurol. 2001;14(6):711-5.

2. Rachinger W, Grau S, Holtmannspötter M, Herms J, Tonn JC, Kreth FW. Serial stereotactic biopsy of brainstem lesions in adults improves diagnostic accuracy compared to MRI only. J Neurol Neurosurg Psychiatry. 2009;80(10):1134-9.

3. Choux M, Lena G, Do L. Brainstem tumors. In: Choux M, Di Rocco C, Hockley A, editors. Pediatric neurosurgery. New York: Churchill Livingstone; 2000. p. 471-89.

4. Jallo GI, Biser-Rohrbaugh A, Freed D. Brain stem gliomas. Childs Nerv Syst. 2004;20(3): 143-53.

5. Zagzag D, Miller DC, Knopp E, et al. Primitive neuroectodermal tumors of the brainstem: investigation of seven cases. Pediatrics. 2000;106(5):1045-53.

6. Dellaretti M, Touzet G, Reyns N, et al. Correlation between magnetic resonance imaging findings and histological diagnosis of intrinsic brainstem lesions in adults. Neuro Oncol. 2012;14 (3):381-5 .

7. Freeman CR, Farmer JP. Pediatric brain stem gliomas: A review. Int J Radiat Oncol Biol Phys. 1998;40(2):265-71.

8. Kaplan AM, Albright AL, Zimmerman RA, et al. Brainstem gliomas in children. A Children's Cancer Group review of 119 cases. Pediatr Neurosurg. 1996;24(4):185-92.

9. Walker DA, Punt JA, Sokal M. Clinical management of brain stem glioma. Arch Dis Child. 1999;80(6):558-64.

10. Fischbein NJ, Prados MD, Wara W, et al. Radiologic classification of brain stem tumors: correlation of magnetic resonance imaging appearance with clinical outcome. Pediatr Neurosurg. 1996;24 (1):9-23.

11. Albright AL, Guthkelch AN, Packer RJ, et al. Prognostic factors in pediatric brain-stem gliomas. J Neurosurg. 1986;65(6):751-5.
12. Bowers DC, Georgiades C, Aronson LJ, et al. Tectal gliomas: Natural history of an indolent lesion in pediatric patients. Pediatr Neurosurg. 2000;32(1):24-9.

13. Koziarski A, Zielinski G, Podgorski JK, Warczynska A. One stage removal of periaqueductal glioma in adult via infratentorial supracerebellar and transaqueductal approaches. Acta Neurochir (Wien). 2004;146(2):169-73.

14. Yeh DD, Warnick RE, Ernst RJ. Management strategy for adult patients with dorsal midbrain gliomas. Neurosurgery. 2002;50 (4):735-8

15. Vandertop WP, Hoffman HJ, Drake JM, et al. Focal midbrain tumors in children. Neurosurgery. 1992;31(6):186-94.

16. Boydston WR, Sanford RA, Muhlbauer MS, et al. Gliomas of the tectum and periaqueductal region of the mesencephalon. Pediatr Neurosurg. 1991-1992;17(5):234-8.

17. Grant GA, Avellino AM, Loeser LJ, Ellenbogen RG, Berger MS, Roberts TS. Management of intrinsic gliomas of the tectal plate in children. Pediatr Neurosurg. 1999; 31:170-6.

18. Kim BS, Jallo GI, Kothbauer K, et al. Chronic subdural hematoma as a complication of endoscopic third ventriculostomy. Surg Neurol. 2004;62:64-8.

19. Chaddad Neto F, Lopes A, Alberto Filho M, Catanoce A, Joaquim AF, Oliveira E. Tectal glioblastoma. Arq Neuropsiquiatr. 2007; 65(4A):996-9.

20. Epstein FJ, Farmer JP. Brainstem glioma growth patterns. J Neurosurg. 1993;78(3):408-12.

21. Tobias ME, McGirt MJ, Chaichana KL, et al. Surgical management of long intramedullary spinal cord tumors. Childs Nerv Syst. 2008;24(2):219-23.

22. Liu Q, Liu R, Kashyap MV, et al. Brainstem glioma progression in juvenile and adult rats. J Neurosurg. 2008;5:849-55. 